\title{
Multilayer Thermionic Refrigeration
}

\author{
G.D. Mahan \\ Department of Physics and Astronomy, \\ University of Tennessee, Knoxville, TN, 37996, and \\ Solid State Division, \\ Oak Ridge National Laboratory, Oak Ridge, TN 37831-6032
}

\begin{abstract}
A review is presented of our program to construct an efficient solid state refrigerator based on thermionic emission of electrons over periodic barriers in the solid. The experimental program is to construct a simple device with one barrier layer using a three layers: metal-semiconductor-metal. The theoretical program is doing calculations to determine: (i) the optimal laver thickness, and (ii) the thermal conductivity.
\end{abstract}

\section{Introduction}

Refrigeration using thermionic emission was proposed in 1994[1, 2]. The original concept had two metal plates separated by a vaccum or air. This device is practical at room temperature only if the metal electrodes have a low work function $(0.3 \mathrm{eV})$ which is below any known value. So the vacuun device is impractical. A related concept is to have a multilayer solid, in which alternte tayers serve as barriers to the flow of electrons $\{3,4,5,6,7]$. Ouly energetic electrons surmount the barriers, which serves as an efficient energy filter. The electrical current is curried by the hot electrons, which leuds to a ligh effective Seebeck coefficient. This concent is called Multi- layer Thermionic Refrigeration.

\section{Experimental Program}

Several other research groups are investigat. ing semiconductor multilayers for use as solid state refrigerators $[8,9,10]$. In these syrstems, the band offsets between successive layers serves as the periodic barriers. Our group has tried an alternate approach of having the multilayers be alternate layers of metals and semiconductors. In this case the Schottky barrier at the metal-semiconductor interface serves as the barrier to the flow of electrons. The semiconductor form the barrier layers.

My experimental colleagues (J. Bates, $G$. Kim, F. Modine, D. Norton, H. Weitering) have been trying to construct simple three layer devices of one semiconductor layers with two metal electrodes. There are several methods of making these thin film devices. Here we list them according to the ease of making films:

1. Sputtering: Satisfactory three layer systems were made by sputtering germanium between two metal electrodes. However: Ge hias too high of a thermal conductivity to demonstrate solid state refrigeration. Numerous attempts were made to sputter binary semiconductors such as $\mathrm{Bi}_{2} \mathrm{Te}_{3}$ 
or PbTe. Sputtering of binaries was unsiccessful. The films were of poor quality and contained large densicies of defects. We are unaware of any inccesstul attempt to create binary films by sputtering.

2. Laser Ablation: ORNL has a major facility in making films by liser ablation. Only oxide materials form will layered hims using this technique. Reports in the literature suggested that $\mathrm{C}_{1,2} \mathrm{O}$ and $\mathrm{BiBaO}_{3}$ both had small values of Schottky bariers with suitable metals. Three layer devices were made of these two materials using laser ablation. Our measturements showed that the resulting Schottky barriers were ton high $(0.7 \mathrm{eV})$ to serve as suitable thermionic retrigerators.

3. Chemical Vupor Deposition: This has not yet been tried.

4. Molecular Beum Epitaxy: Weitering and Kim have constructed an MBE machine to make films for thermionic refrigerators. Weitering is giving a talk on this work at this meeting.

So far the experimental work has been inconclusive. No device has yet been made which demonstrate efficient coolins.

\section{Theoretical Considera- tions}

\subsection{Layer Thickness}

One crucial issue for the device efficiency is to select the appropriate thickness for the barrier layer. The proper choice depends upon ones view of how the device operates. Our view is that efficient cooling requires that the electrons ballistically traverse the barrier layer. Then there is a requirement that the lavers must be thinner then the mean-free-path of the electron $\left(L<B_{p}\right)$.

There are several definitions of mean-freepath (mfp): for momentum relaxation $\ell_{p}$, for energy relaxation $\ell_{E}$ and for phase breaking $\ell_{D}$. Generally $\ell_{E}>>\ell_{p}$. We believe that the relevant $\mathrm{mfp}$ to determine the barrier thickness is the one for momentum relaxation $\ell_{p}$. We have performed calculations at room temperature $(T=300 k)$ of the $\mathrm{mfp} \varepsilon_{p}$ for some common n-type semiconductors which have a single conduction bund ellipsoid. The mean-kreepath was limited by scattering by phonons and impurities. The phonon scattering gave values of the mfp on the order of micrometers. The scattering by impurities gave values of the $\mathrm{mfp}$ on the order of $10-100 \mathrm{~nm}$. Sinc impurity scattering gives the small value, it dominates the answer. The most probable value of $\ell_{\rho} \sim 5 a_{g}^{*}$, where $a_{\beta}^{*}$ is the effective Botr radius of an electron bound to a donor in the semiconductor. This result is remarkably independent of the concentration of impurities.

Similar calculations have been done for the $m f p$ of phonons, and for the distribution of mfps. Some results will be shown.

\subsection{B-Factor}

In thermoelectrics the B-Factor is a dimensionless number (called $B_{T E}$ ) which determines the efficiency of thermoelectrics(11). Vining and Mahan[12] showed that there is an equivalent $B$-Factor for thermionic refrigeration (called $B_{T f}$. These two are

$$
\begin{aligned}
B_{T I} & =\frac{m^{*} k_{B}\left(k_{B} T\right)^{2} L}{2 \pi^{2} \hbar^{3} K_{L}} \\
B_{T E} & =\frac{m^{-} k_{B}\left(k_{B} T\right)^{2} \ell_{p}}{2 \pi^{3 / 2} \hbar^{3} K_{S L}} \\
\theta_{p} & =\tau v_{p}=\tau \sqrt{\frac{2 k_{G} T}{m^{*}}}
\end{aligned}
$$




$$
\frac{B_{T I}}{B_{T E}}=\frac{L}{\ell_{p} \sqrt{\pi}}
$$

and $b=e \phi / k_{B} T$ where eo is the work function in thermionic emission. Given that $L<\ell_{p}$ according to our prior discussion, then the Bfactor $\left(B_{T I}\right)$ for thermionic emission is always less than that for thermoelectrics. Since the larger $B$ gives the larger efficiency[1 I], then a thermoelectric device is more efficient than a thermionic device if they lave the same thermal conductivity.

\subsection{Thermal Conductivity}

All solid state refrigeraturs require a low value of the thermal conductivity for efficient operation[11]. It is possible that a multilayer system has a lower thermal conductivity than a pure material[8, 13,14,15.16,17,18,19,20]. This result has been found in early experi. ments, and has been predicted by cislculations. Here we confine the discussion to thermal conductivity along the $c$-axis, which is perpendicular to the plane of the layers. We have done calculations which show thit, there is an optimal thickness of the layers in order to have a minimum thermal conductivity. In this case the important parameter. which determines the minimum layer thickness, is the phonon mfp. There are two different methods of calculating the thermal conductivity of superlattices, and they give dramatically different answers. They are the classical or particle theory, and the wave theory.

The classical theory includes the interface boundary resistance[21]. A superlattice with alternating layers of thickne'ss $L_{1,2}$ has a thermal resistance for one repeat unit

$$
\begin{aligned}
& R_{S L}=L_{1} / K_{1}+L_{2} / K_{2}+2 R_{B} \\
& K_{S L}=\frac{2 L}{R_{S L}}=\frac{2 L}{L\left(1 / K_{1}+1 / K_{2}\right)+2 R_{B}}(5)
\end{aligned}
$$

where $K_{j}$ is thermal conductivity of the individual layers. and $R_{B}$ is the thermal boundary resistance. Assume that $L_{1}=L_{2} \equiv L$. The effective thermal conductivity of the superlattice is $K_{S L}$. This classical prediction is that the thermal conductivity decreases as the laver thickness $L$ decreases[20]. The same result is obtained by solving the Boltzmann equation for the transport. Boltzmann equation treats the phonons as particles which scatter from the boundaries. Wave phenomena is ignored.

The wave theory calculates the actual phonon modes $\omega_{\lambda}(\mathbf{k})$ of the superlattice, where $\lambda$ is the band index. They are used to calculate the thermal conductivity from the usual formula in $d$-dimensions[22]

$K(T)=\sum_{\lambda} \int \frac{d^{d} k}{(2 \pi)^{d}} \hbar \omega_{\lambda}(\mathrm{k})\left|v_{z}(\mathrm{k})\right| \ell(\mathrm{k}) \frac{\partial n(\omega, T)}{\partial T}$

where $\ell(\mathbf{k})$ is the phonon mfp and $n(\omega, T)$ is the Bose-Einstein distribution function. At high temperatures, approximate $n \sim$ $k_{B} T / \hbar \omega_{\lambda}(k)$ which gives

$$
K(T)=k_{B} \sum_{\lambda} \int \frac{d^{d} k}{(2 \pi)^{d}}\left|v_{z}(\mathrm{k})\right| \ell(\mathrm{k})
$$

The above formula is quite general. Two important special cases of constant relatiation time $\left(K_{T}\right)$ and constant $m f p\left(K_{\ell}\right)$ are

$$
\begin{aligned}
& K_{T}(T)=k_{B} T \sum_{\lambda} \int \frac{d^{d} k}{(2 \pi)^{d}} v_{z}(k)^{2} \\
& K_{\ell}(T)=k_{B} \ell \sum_{\lambda} \int \frac{d^{d} k}{(2 \pi)^{d}}\left|v_{z}(\mathrm{k})\right|
\end{aligned}
$$

Wave interference leads to band folding[23, 24], which leads to a reduction of the phonon velocities. Both $K_{T}$ and $K_{\ell}$ are reduced by wave interference.

These assertions are best illustrated in one dimension, for the atomic chain. Our model[25] for a superlattice has all spring constants identical, and the layers differ in their 

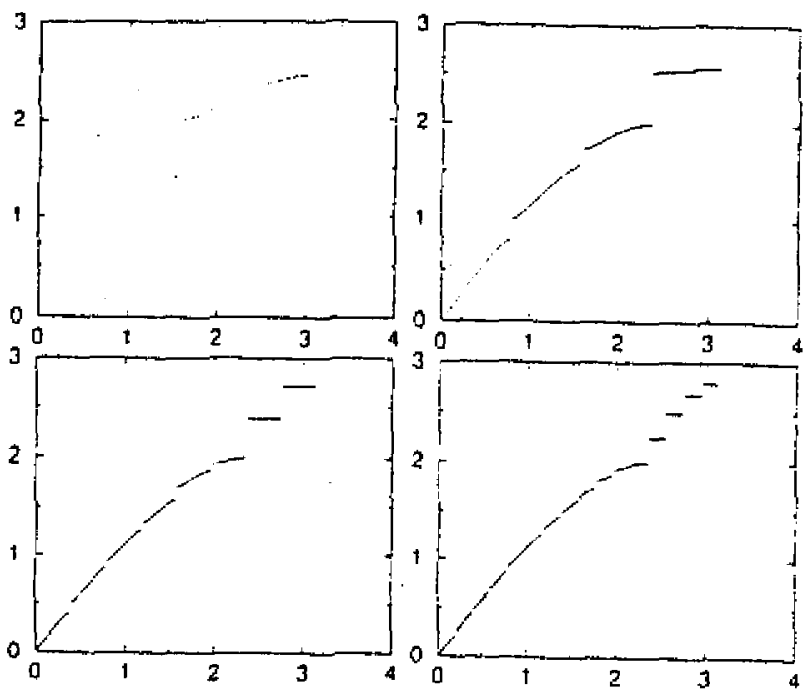

Figure 1: Spectrum of $N=2,4,8,16$ superlattices with mass ratio $\alpha=2$ in the extended zone representation.

masses. For a repeat distance of $N$ - lizyers, one layer has $N / 2$ atoms of mass $m_{1}$ and the other layer has $N / 2$ atoms of mass $m_{2}=m_{1} / \alpha$. The characteristic equation $k(\omega)$ of the normal modes is [25]

$$
\begin{aligned}
& \cos (k N)=\cos \left(k_{1} N / 2\right) \cos \left(k_{2} N / 2\right)-(10) \\
& \frac{1-\cos \left(k_{1}\right) \cos \left(k_{2}\right)}{\sin \left(k_{1}\right) \sin \left(k_{2}\right)} \sin \left(k_{1} N / 2\right) \sin \left(k_{2} N\right.
\end{aligned}
$$

$$
\text { ) }
$$

where $\cos \left(k_{1}\right)=1-\omega^{2} / 2, \cos \left(k_{2}\right)=1-$ $\omega^{2} /(2 \alpha)$ define the wave vectors $\left(k_{1} . k_{2}\right)$ in the individual layers in dimensionless units. A typical spectrum is shown in Fig.1, where $\alpha=2$ and the SL periods are $N=2,4,8,16$. Modes with frequency $\omega>2$ are confined within the layer of the lighter atom, and contribute little to the thermal conduction. As the value of $N$ is increased in Fig. 1, there is more band folding. and the average velocity decrenses. Fig.2 shows the thermal conductivity as a function of superlattice period $[25,26,27]$ The result for constant $\mathrm{mfp}$ is normalized to $k_{B} \ell \omega_{2, \max }$, where wam $=2$ is the maximum phonon Erequency in the layer 1 of heary mass. The heat $T$ his result is for one dimension. Similar curves are found for every casse which we have

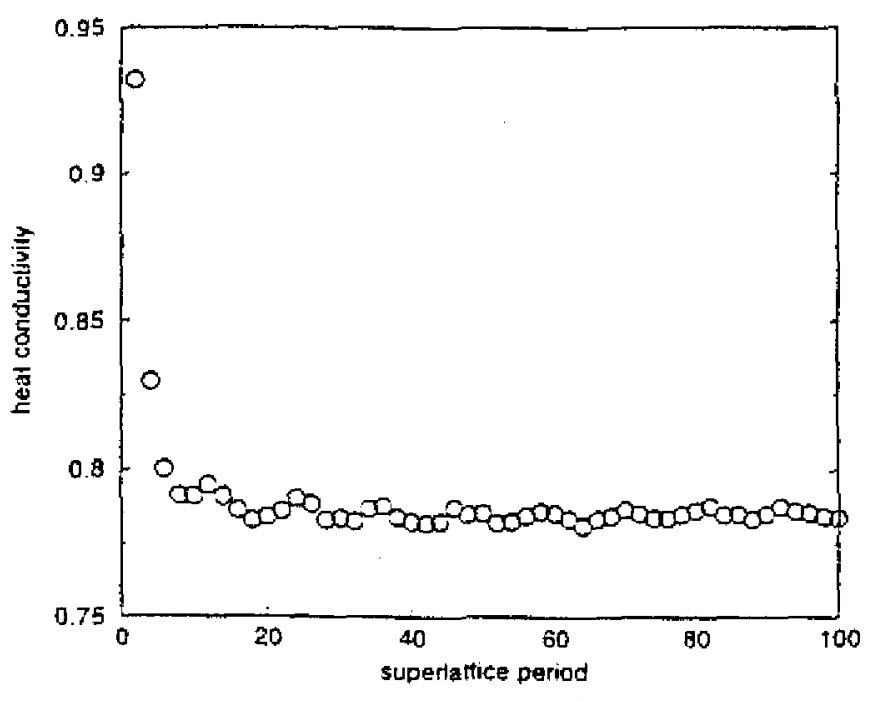

Figure 2: Heat conductivity in one dimension as a function of superlattice period for mass ratio $\alpha=2$. Dimensionless units found by dividing (11) by $2 k_{B} \ell$.

calculated: for different values of mass ratio $\alpha$, and for both $K_{\ell}$ and $K_{+}$. Generally, increasing $N$ : (i) increases the amount of band folding, (ii) decreases the average velocity in the SL, and (iii) decreases the thermal conductivity. Similar calculations of $K_{t}$ in two and three dimensions show the same tesult that the thermal conductivity declines with increasing $N$, for all values of $\alpha[25]$.

The two methods of calculation of the thermal conductivity, by boundary resistance and by band folding, give opposite predictions for the thermal conductivity as a function of layer thickness. The two viewpoints are reconciled by including the mfp in the calculations. When the layer thickness exceeds the mfp. then inter ference effects should diminish, and the particle model should become applicable. The wave model should apply when $L<\ell$ and the particle model should apply when $L>\ell$. The mfp $\ell$ is audded to the calculation of the heat transport by including a complex part to the wave vectors $k_{1,2}$, which is $i / \ell$. The properties of the superlattice are calculated using eqn.(10)but now including the imaginary part 


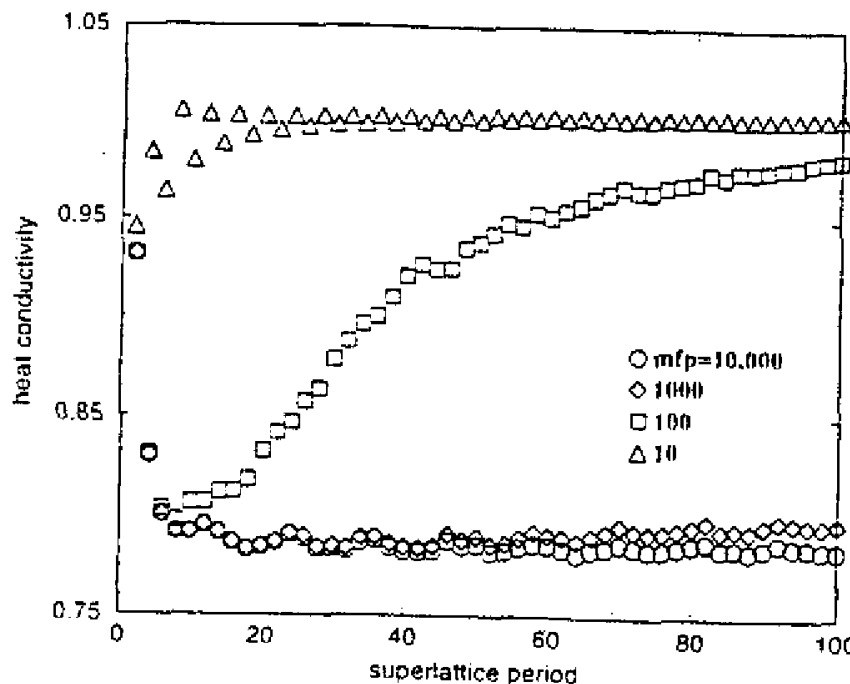

Figure 3: Thermal conductivity in one dimension as a function of superlattice period for mass ratio $\alpha=2$ for different values of the phonon $\mathrm{mfp}$ which is given in units of lattice periods.

of $k_{1,2}$. Making $k_{1.2}$ compiex makes $k$ complex, and the effective wave vector is its real part. The band folding is found to diminish as the mfp is decreased for a fixed value of $N[25]$.

Fig. 3 shows the thermal conductivity in one dimension as a function of SL period for four different values of $\mathrm{mfp}$ and $\alpha=2$. The $\operatorname{mfp}$ is given in terms of the number of lattice spacings (note: not SL spacings). Sirnilar curves are found for other values of $\alpha$. For large values of $\ell$ the results are identical to figure 2 (which had $\ell=\infty$ ). For small values of $\mathrm{mfp}$ $(\ell=10)$ the thermal conductivity is nearly independent of SL period, except where it falis at small values of $N$. This reduction is probabiy due to thermal boundary resistance. Using eqn.(1) shows a very small vitue of boundary resistance $R_{B}$. For $\ell=100$ the thermal conductivity falls as $N$ increases, reaches a minimum, and then starts to increase. This latter behavior is the situation expected in the experiments. At room temperature, in most solids, anharmonic scartering limits the phonon mpf to value in the range of 10-100 lattice con- stants, which is also the typical value of SL parameter in current devices. Therefore we expect the experimental thermal conductivities to behave as the curve marked $\ell=100$ in fig.3. The thermal conductivity should have a minimum value when plotted vs. SL period. The minimum occurs at the cross over between the particle and wave-interference types of transport. One experimental result has this behavior[8].

\section{Conclusion}

We have considered the experimental variables which determine the optimal operation of a multilayer refrigerator based upon thermionic emission. The barrier thickness will be determined by the minimum in the thermal conductivity, which is determined by the phonon $\mathrm{mfp}$. The phonon $\mathrm{mfp}$ is generally shorter than the mfp of the electron. The optimal barriers thicknesses will be quite short, on the order of nanometers rather than hundreds of nanometers. This value is much shorter than the $\mathrm{mfp}$ of the electron, so that the electrons are safely in the ballistic regime. For these very short barriers the multilayer thermionic refrigerator may not be very efficient, since its B-factor is relatively small.

\section{Acknowledgements}

Research support is acknowledge from the Office of Naval Research Contract No. No001498-1-0742, from the University of Tennesee. and from Oak Ridge National Laboratory managed by Lockheed Martin Energy Research Corp. for the U.S. Department of Energy under contract DE-AC05-96OR22464. 


\section{References}

(1) G.D. Mahan, J. Appl. Phys. 76, 4362 (1994)

[2] G.S. Nolas and H.J. Goldsmid, J. Appl. Phys. 85, 4060 (1999)

[3] L.W. Whitlow and T. Hirano, Proceedings of the 12 International Conference on Thermoelectrics (unpublished) 1993. p. 39

(4) D.M. Rowe and G. Min, Proceedings of the 13 International Conference on Thermoelectrics (unpublished) 1994: p. 339

[5] G.D. Mahan and L.M. Woods, Phys. Rev. Lett. 80, 4016 (1998)

(6) G.D. Mahan, J.O. Sofo and M. Bartkowiak, J. Appl. Phys. 83, 4683 (1998)

[7] R. Zhou, D. Dagel and Y.H. Lo, Appl. Phys. Lett. 74, 1767 (1999)

[8] R. Venkatasubramanian and T. Colpitts, in Thermoelectric Materials-New Directions and Approaches. ed. T.M. Tritt, M.G. Kanatzidis, H.B. Lyons $\mathrm{Jr}$, and G.D. Mahan (Materials Research Society, 1997) Vol. 478, pg. 73

[9] A. Shakouri and J.E. Bowers, Appl. Phys. Lett. 71,1234 (1997)

\{10| A. Shakouri, C. LaBounty, J. Piprek, P. Abraham, J.E. Bowers, Appl. Phys. Lett. 74. 88 (1999)

[11] G. D. Mahan, in Solid State Physics, ed. H. Ehrenreich and F. Spaepen (Academic Press, 1998) Vol. 51, pg 81

(12) C.B. Vining and G.D. Mahan, J. Appl. Phys, (in press)
[13] T. Yao, Appl. Phys. Lett. 51, 1798 (1987)

(14) G. Chen, C.L. Tien, X. Wu and J.S. Smith, J. Heat transfer 116, 325 (1994)

(15] P. Hyldgaard and G.D. Mahan, Proc.Int. Conf. on Thermal Conductivity: Nashville, (November, 1995)

[16] W.S. Capinski and H.J. Maris, Physica B 219-220, 699 (1996)

(17) S.M. Lee, D.G. Cahill, R. Ventakasubramanian, Appl. Phys. Lett. 70, 2957 (1997)

[18] G. Chen and M. Neagu, Appl. Phys. Lett. 71,2761 (1997)

[19] P. Hyldgaard and G.D. Mahan. Phys. Rev. B 56, 10754 (1997)

[20] G. Chen, Phys. Rev. B 57, 14958 (1998)

[21] E.T. Schwartz and R.O. Pohl, Rev. Mod. Phys. 61, 605 (1989)

[22] J. Ziman Electrons and Phonons (Cambridge University Press, 1960)

[23] S.Y. Ren and J.D. Dow, Phys. Rev. B 25, 3750 (1982)

[24] B.K. Ridley, Electrons and Phonons in Semiconductor Multilayers (Cambridge. 1997)

[25] M.V. Simkin and G.D. Mahan (submitted)

[26] P. Hyldgaard (unpublished)

[27] S. Tamura, Y. Tanaka, and H.J. Maris, preprint 